\title{
A caveat about applications of the unilateral Fourier transform
}

\author{
Antonio S. de Castro*1] \\ ${ }^{1}$ Universidade Estadual Paulista "Júlio de Mesquita Filho", Departamento de Física, Guaratinguetá, SP, Brasil.
}

Received on April 07, 2020. Accepted on May 23, 2020.

\begin{abstract}
It is presented a warning about the erroneous use of unilateral Fourier transform with nonhomogeneous Dirichlet or Neumann boundary conditions in a well-known textbook on integral transforms, and also in a few papers recently diffused in the literature.
\end{abstract}

Keywords: unilateral Fourier transform, Fourier sine transform, Fourier cosine transform.

Integral transforms are used in a variety of applications, for example, to evaluate certain definite integrals, to transform a partial differential equation into an ordinary differential equation, to transform an ordinary differential equation into a simpler differential equation or into an algebraic equation, and they also can play a more theoretical role in applied problems.

L. Debnath and D. Bhatta wrote in the Preface to the Second Edition of their book [1]: "When the first edition of this book was published in 1995 under the sole authorship of Lokenath Debnath, it was well received, and has been used as a senior undergraduate or graduate level text and research reference in the United States and abroad for the last ten years." It is really a wellknown book, now in its third edition [2]. Unfortunately, it is a book marked by great carelessness concerning the unilateral Fourier transform and its applications which has apparently triggered the emergence of a number of papers [3]- 11] emulating the misuses found there. Truthfully, one of the cited papers not only emulates but expands the misguided applications $[10$.

The purpose of the present work it to call attention to the catastrophic embroilments mentioned above in order to keep students on alert. It is also a warning to reduce their own risk making future misleading research efforts. Even undergraduate students can follow the argument easily.

The Fourier sine and cosine transforms of $f(x)$ are denoted by $\mathcal{F}_{s}\{f(x)\}=F_{s}(k)$ and $\mathcal{F}_{c}\{f(x)\}=F_{c}(k)$, respectively, and are defined by the integrals (see, e.g. [12]- 13])

$$
\begin{aligned}
& F_{s}(k)=\mathcal{F}_{s}\{f(x)\}=\sqrt{\frac{2}{\pi}} \int_{0}^{\infty} d x f(x) \sin k x, \\
& F_{c}(k)=\mathcal{F}_{c}\{f(x)\}=\sqrt{\frac{2}{\pi}} \int_{0}^{\infty} d x f(x) \cos k x,
\end{aligned}
$$

*Correspondence email address: antonio.castro@unesp.br. where $k \geq 0$. The original function $f(x)$, based on certain conditions, can be retrieved by the inverse unilateral Fourier transforms $\mathcal{F}_{s}^{-1}\left\{F_{s}(k)\right\}$ and $\mathcal{F}_{c}^{-1}\left\{F_{c}(k)\right\}$ expressed as

$$
f(x)=\sqrt{\frac{2}{\pi}} \int_{0}^{\infty} d k F_{s}(k) \sin k x,
$$

and

$$
f(x)=\sqrt{\frac{2}{\pi}} \int_{0}^{\infty} d k F_{c}(k) \cos k x .
$$

Sufficient conditions for the existence of the above integrals are ensured if $f(x), F_{s}(k)$ and $F_{c}(k)$ are absolutely integrable.

The use of integral transforms is worthless if their inversion formulas fail. The behaviors of $f(x)$ and $d f(x) / d x$ at the origin have been belittled in Ref. 11 and in a number the recent papers diffused in the literature regarding the unilateral Fourier transforms. It is essential to note that equation (3) dictates that the Fourier sine transform is invertible only if $\left.f(x)\right|_{x=0}=0$ (homogeneous Dirichlet boundary condition), whereas equation (4) is decided upon $d f(x) /\left.d x\right|_{x=0}=0$ (homogeneous Neumann boundary condition). The convenience of using the sine or cosine transform is dictated by those homogeneous boundary conditions. Therefore, a smart use of the unilateral Fourier transform should pay attention to the different homogeneous boundary conditions at the origin.

In Sec. 2.13 of Ref. [1], the authors went for the straightforward approach putting (3) and (4) as definitions of the inverse Fourier sine and cosine transforms, respectively. In the Example 2.13.1, they presented the results for $\mathcal{F}_{c}\left\{e^{-a x}\right\}$ and $\mathcal{F}_{s}\left\{e^{-a x}\right\}$ with $a>0$ and used the inversion formulas to calculate others transforms. Nevertheless, $e^{-a x}$ does not have the mandatory behaviors at 
the origin. The readers should note that the expression

$e^{-a x}=\frac{2 a}{\pi} \int_{0}^{\infty} \frac{\cos k x}{k^{2}+a^{2}} d k=\frac{2}{\pi} \int_{0}^{\infty} \frac{x \sin k x}{k^{2}+a^{2}} d k, \quad a>0$

is not in conformity with truth because $\left.e^{-a x}\right|_{x=0}=1$ and $d e^{-a x} /\left.d x\right|_{x=0}=-a$.

In Example 2.15.1 of Ref. [1], the authors considered the one-dimensional diffusion equation on a half line $(0<x<\infty)$ encompassing nonhomogeneous Dirichlet or Neumann boundary conditions at the origin. They denoted the Fourier sine transform of $u(x, t)$ with respect to $x$ by $U_{s}(k, t)$ and arrived at

$$
U_{s}(k, t)=\sqrt{\frac{2}{\pi}} \int_{0}^{\infty} u(x, t) \sin k x d x
$$

but the inversion of $U_{s}(k, t)$

$$
u(x, t)=\sqrt{\frac{2}{\pi}} \int_{0}^{\infty} U_{s}(k, t) \sin k x d k
$$

as we already know, demands $u(0, t)=0$ in such a way that only the homogeneous Dirichlet boundary condition is allowed. In fact, with $u(x, 0)=0$ and $u(0, t)=f(t)$, the authors found (see Eq. 2.15.8 in Ref. [1])

$$
u(x, t)=\frac{x}{4 \sqrt{4 \pi \kappa}} \int_{0}^{t} f(\tau) \exp \left[-\frac{x^{2}}{4 \kappa(t-\tau)}\right] \frac{d \tau}{(t-\tau)^{3 / 2}}
$$

where $\kappa$ is a constant. Clearly, equation 8 is not in agreement with the nonhomogeneous Dirichlet boundary condition $u(0, t)=f(t)$.

In Example 2.15.2 of Ref. 1], the authors went ahead and considered the Laplace equation in the quarter plane $(0<x<\infty, 0<y<\infty)$ with the boundary conditions $u(0, y)=a$ and $u(x, 0)=0$, where $a$ is a constant. They applied the Fourier sine transform with respect to $x$ and succeeded in reaching the formal solution

$$
u(x, y)=\frac{2 a}{\pi} \int_{0}^{\infty} \frac{1}{k}\left(1-e^{-k y}\right) \sin k x d x
$$

Can we sincerely see here the nonhomogeneous Dirichlet boundary condition $u(0, y)=a$ ?

For short, a fruitful use of the Fourier sine and cosine transforms demands maximal attention given to the behavior at the origin. Apparently influenced by a wellknown textbook, that care has been neglected by some authors in recent times.

\section{Acknowledgments}

This work was supported in part by means of funds provided by Conselho Nacional de Desenvolvimento Científico e Tecnológico (CNPq), Brazil, Grant No. 09126/20193 (PQ).

\section{References}

[1] L. Debnath and D. Bhatta, Integral Transforms and Their Applications, (Taylor and Francis Group, London, 2007), 2nd ed.

[2] L. Debnath and D. Bhatta, Integral Transforms and Their Applications, (Taylor and Francis Group, London, 2015), 3rd ed.

[3] C.Rubio-Gonzalez and J.J. Mason, J. Appl. Mech. 66, 485 (1999).

[4] C. Rubio-Gonzalez and J.J. Mason, Comput. Struct. 76, 237 (2000).

[5] C. Rubio-Gonzalez and J.J. Mason, Int. J. Fracture 108, 317 (2001).

[6] E. Lira-Vergara and C. Rubio-Gonzalez, Int. J. Fract. 135, 285 (2005).

[7] C. Rubio-Gonzalez and E. Lira-Vergara, Int. J. Fract. 169, 145 (2011).

[8] M. Nazar, M. Zulqarnain, M.S. Akram and M. Asif, Commun.Nonlinear Sci. Numer. Simulat. 17, 3219 (2012).

[9] N. Shahid, M. Rana and I. Siddique, Bound. Value Probl. 48, 1 (2012)

[10] J.C. de Araújo and R.G. Márquez, Rev. Eletr. Paul. Mat. 11, 136 (2017).

[11] J.C. de Araújo and R.G. Márquez, TEMA 20, 95 (2019).

[12] E. Butkov, Mathematical Physics (Addison-Wesley, Reading, 1968).

[13] I.S. Gradshteyn and I.M. Ryzhik, in: Table of Integrals, Series, and Products, edited by A. Jeffrey and D. Zwillinger (Academic Press,New York, 2007), 7th ed. 